\title{
Extraordinary Transmission of Three-Dimensional Crescent-like Holes Arrays
}

\author{
Yang Shen • Mingkai Liu • Jensen Li • Xia Chen • \\ Hai-Xia Xu • Qiangzhong Zhu • Xuehua Wang • \\ Chongjun Jin
}

Received: 5 June 2011 / Accepted: 17 October 2011 / Published online: 22 November 2011

(C) The Author(s) 2011. This article is published with open access at Springerlink.com

\begin{abstract}
We developed a method to fabricate a periodic array of three-dimensional crescent-like holes (3DCLH) via an inverted hemispherical colloidal lithography. It is found that there exists an extraordinary optical transmission in this non-planar perforated periodic array of 3DCLH when the electric field of the incident light is perpendicular to the cross-line of the crescent-like hole. This extraordinary optical peak is insensitive with the incident angles and sensitive with the angle between the electric field of the incident light to the cross-line of the 3DCLH. Numerical simulation based on finite-difference time-domain method reveals that this peak is caused by an asymmetric localized surface plasmon resonance. This structure might be useful for the optical sensing and optical-integrated circuits.
\end{abstract}

Keywords Surface plasmon polariton · Localized surface plasmon $\cdot$ Extraordinary optical transmission $\cdot$ Colloidal lithography

\section{Introduction}

Extraordinary optical transmission (EOT) of light passing through sub-wavelength holes array in a metallic film has

Y. Shen $\cdot$ M. Liu $\cdot$ X. Chen $\cdot$ H.-X. Xu $\cdot$ Q. Zhu $\cdot$ X. Wang $\cdot$

C. Jin $(\triangle)$

State Key Laboratory of Optoelectronic Materials

and Technologies, School of Physics and Engineering,

Sun Yat-Sen University,

Guangzhou 510275, China

e-mail: jinchjun@mail.sysu.edu.cn

J. Li

Department of Physics and Materials Science,

City University of Hong Kong,

Hong Kong, China been found in 1998 [1]. It has attracted lots of attention, because this effect can be used in sub-wavelength integrated optical circuits [2-7], biosensors [8], surface plasmon enhanced Raman scattering [9, 10]. Lots of efforts have been made on this field in order to understand the fundamental physical mechanism and design more efficient structures [11-26]. Nowadays, it is considered that EOT of the sub-wavelength holes array is caused by the coupling between the incident waves and the surface plasmon polariton (SPP) with the help of the periodic twodimensional holes array [7, 11]. In addition to circular holes arrays, EOT phenomenon was also found in holes arrays with different shapes, such as elliptical, rectangular, and annular holes [12-18]. Later, it is found that the localized surface plasmon resonance (LSP) could also play a role in the EOT in the holes with rectangular or elliptical shapes together with the SPP. To specify the contribution of the LSP, arrays of holes with a nanoparticle in each hole are fabricated and measured [19-22], it is found that both the SPP and the LSP contribute to the optical transmission, and both the real and imaginary parts of the polarizability of the particles which induce LSP play important roles in the transmission suppression and enhancement [20]. Very recently, an array of holes with very acute angles was found to exhibit a strong EOT effect caused by the LSP [27], an array of three-dimensional holes was also found that it has strong EOT effect induced by the LSP [28]. The waveguide mode across each hole can also play an important role in the extraordinary transmission through Fabry-Perot resonance [14, 29]. Until now, there have been no reports about the extraordinary transmission in a non-planar asymmetric structure. Here, we developed a unique fabrication method and prepared periodic arrays of three-dimensional crescentlike holes (3DCLH) as shown in Fig. 1a, by colloidal 


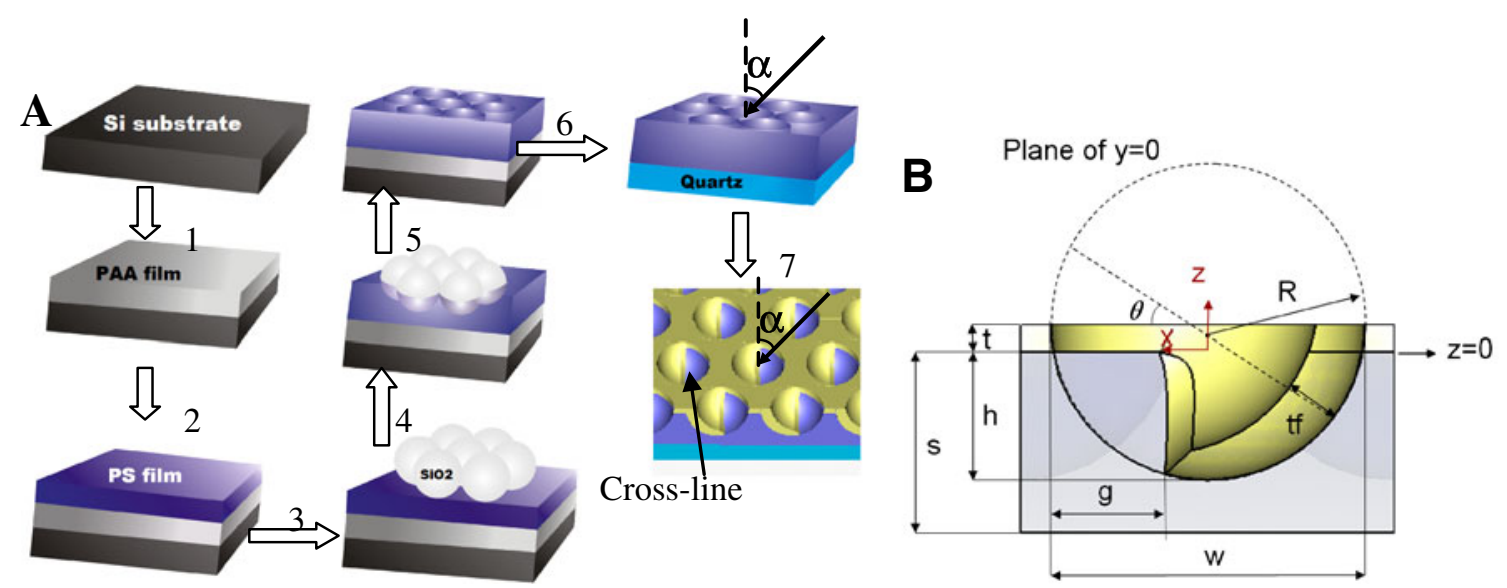

Fig. 1 a The fabrication procedure for a periodic array of threedimensional crescent-like holes. 1 Spin-coating of sacrificial polyacrylic acid $(P A A)$ film on silicon; 2 Spin-coating a layer of polystyrene $(P S) ; 3$ Spin-coating a multilayer of silica spheres with a diameter of $264 \mathrm{~nm}$; 4 Heating the sample to let silica sphere embed into the PS film; 5 Removing the embedded silica particles by HF

lithography with shadow metal evaporation method. We report, to our knowledge, the first evidence of the extraordinary transmission induced by an asymmetric localized surface plasmon resonance in the arrays of 3DCLHs. This phenomenon can be useful for the coupling of the incident light to localized surface plasmon, e.g., for sensing applications. It is also valuable in the applications of sub-wavelength optoelectronic circuits.

Colloidal lithography has been widely exploited in the fabrication of surface plasmonic structures. By using two-dimensional array of colloidal spheres as templates, metal crescents, discs, holes, and split-ring resonators have been prepared after the various metal evaporation processes and reactive-ion etching procedure [30-34]. By utilizing thermal annealing to shape the interstitial holes of close-packed monolayer spheres, metal nano-ring, splitring, and nano-disc can also be achieved without the reactive-ion etching process $[35,36]$. Although colloidal nanospheres can directly form a template on the flat substrate, a large area of periodic monolayer spheres is usually hard to be achieved. Until recently, a method to introduce 2D nanostructure into the polymer film via colloidal lithography was reported [37, 38]. In this method, through thermal annealing or imprinting process, only the top or bottom layer of the spheres can be transferred to the polymer and form a large area of periodic array of monolayer spheres. It has been used to fabricate nano-metal shells, nano-dimples [37], nanograils [39], and nanodiscs [40]. Here, based on our previous work on fabricating nanodiscs [40], we have developed a method to prepare a periodic array of three-dimensional crescent-like holes. solution; 6 The PAA film was dissolved in water, and a floating PS film with hemispherical dimple array was transferred to quartz slide; 7 A shadow metal evaporation method was employed to side deposit a layer of silver. b Cross-section view of the unit cell cutting along a line which passes through the center of the circle and perpendicular to the cross-line

\section{Fabrication of 3D Crescent-like Holes Array}

To fabricate 3D crescent-like holes array, an inverted hemispherical colloidal lithography method [40] is adopted; the fabrication procedure plotted in Fig. 1a can be described as follows: First, a layer of sacrificial polyacrylic acid (PAA) film was spin-coated on a cleaned silicon wafer, and baked at a hotplate with $120^{\circ} \mathrm{C}$ for 5 min - the thickness of the PAA is around $220 \mathrm{~nm}$; then, a layer of $137 \mathrm{~nm}$ polystyrene (PS) film was spin-coated on the sacrificial layer, the baking temperature and time were $120^{\circ} \mathrm{C}$ and 5 min respectively; after that, the surface was modified by oxygen etching. Secondly, a multilayer of silica spheres with a diameter of $264 \mathrm{~nm}$ was spin-coated on the PS film, then the sample was placed on the hotplate with a temperature of $115^{\circ} \mathrm{C}$ for $7 \mathrm{~min}$ to make the bottom layer of silica spheres embed into the PS film with a depth of around $98 \mathrm{~nm}$. Thirdly, the silica spheres were removed by hydrofluoric acid (HF) with a concentration of $2 \%$, a layer of dimple array was formed on the PS film, and then the PAA film was dissolved in water, a floating patterned PS film was formed and transferred onto the quartz slide. Finally, a shadow evaporation method was employed to deposit a layer of silver on the patterned PS film, a 3D crescent-like holes array was formed. The schematic structure of fabricated sample is shown in the last step of Fig. 1a. On the top surface, it looks like a periodic array of holes. However, within a unit cell, part of dimple is also deposited with silver because the shadow evaporation method can deposit metal on the part of the dimple by varying the deposition angle $\alpha$, as shown in Fig. 1a. In other words, how much area within the dimple is deposited 
with silver can be controlled by $\alpha$. It looks like an array of crescent holes in top view; it is a three-dimensional structure. Here, it is named as a periodic array of threedimensional crescent-like holes (3DCLH). The thicknesses of the silver at the top surface and the dimples are quite different since the metal evaporation angle varies at different parts within a unit cell. Figure $1 b$ is the crosssectional view of the unit cell by cutting along a line which passes through the center of the circle and is perpendicular to the cross-line. $t$ is the thickness of the metal on the top of the PS film, $\theta$ is the metal evaporation angle, $g$ is the gap width of the crescent-like hole, $t f$ is the thickness of the thickest metal part where silver is deposited vertically. The interface between the PS top surface and metal layer is at $z=0$. The depth of the dimple before depositing metal layer is $h$. The diameter of the dimple is $w$.

Scanning electron microscope pictures of the samples are shown in Fig. 2. Figure 2a, b shows two periodic arrays of 3DCLHs, where black area is not covered with silver. The thicknesses of the silver at top plates in Fig. 2a and b are 55 and $37 \mathrm{~nm}$, respectively. The diameter of the upper circles and the depth of the dimples before silver evaporation are about 255 and $98 \mathrm{~nm}$, respectively. The period of the triangular structure is $283 \mathrm{~nm}$. The area covered with silver is different for different samples. Here, we define $f$ as a ratio of area without silver to the total area in the twodimensional plane view. It should be noted that, due to the nature of colloidal lithography, the pattern is composed of many single domains in different orientations with maximum size of $10 \times 10 \mu \mathrm{m}^{2}$.

\section{Optical Measurement and Analysis}

To characterize the optical properties of 3DCLHs, we measured the zero-order angle-resolved transmission for $\mathrm{p}$ polarized waves (the electric field is on the incident plane). It should be pointed out that, in all the angle-resolved spectra shown in this paper, the curves are plotted with subsequent vertical offsets of 5\% for each step increase of incident angle. Figure $3 \mathrm{a}$ shows the results where the electric field is pointing along with the cross-line of the $3 \mathrm{DCLH}$ as shown in the inset. Two transmission dips are in 546 and $634 \mathrm{~nm}$ at normal incidence, as indicated by red arrows; these two dips are composed of a large dip. There are two peaks which are at 570 and $1,028 \mathrm{~nm}$. In order to understand this transmission, we simulated the transmission of the 3DCLHs arrays via finite-difference time-domain (FDTD) method [41]. Because the domain size is about $10 \mu \mathrm{m}$, the cross-lines of 3DCLHs are oriented to different direction from $\Gamma-M$ to $\Gamma-K$ in various domains with different lattice orientations. In simulation, we have to consider all these conditions. Here, we simulated various 3DCLHs arrays with their cross-lines oriented to different lattice directions, and made an average of these transmissions. The simulated transmission spectra of the 3DCLHs arrays with their cross-line parallel to $\Gamma-M$ and $\Gamma-K$ directions are nearly the same except that there is a surface plasmon polariton peak at $490 \mathrm{~nm}$ for $\Gamma-M$ direction, as shown in Fig. 3b. This means that the different orientations of the crescent-like holes arrays have the same optical transmission when the wavelength is larger than $550 \mathrm{~nm}$. In other words, when the wavelength is larger than $550 \mathrm{~nm}$, the transmission property of the array are mainly decided by 3DCLH rather than the periodic structure. The simulation results show that there are two dips at 554 and $668 \mathrm{~nm}$, and two peaks at 600 and $952 \mathrm{~nm}$ as well. Overall, the simulation results match with experimental results well, except that there exists a deviation at peak position which may be caused by the structure used in simulation not being perfectly the same as the real structure (the rough surface is not considered here). We further inspect the electric field distribution at each dips and peaks, as shown in Fig. 4a-d. It is found that, at the peak position of $962 \mathrm{~nm}$, the electric field is concentrated on the outer surface of 3DCLH and strongly localized at the crescent tip, which enables the resonant field to be re-radiated into the PS film as transmitted wave. In a unit cell, the resonant mode is a dipole resonance, as indicated by the charge polarity. The strong localized field at the crescent tip is mainly caused by
Fig. 2 Scanning electron microscope pictures of 3DCLHs, where black area is not covered with silver. a 3DCLH with $55 \mathrm{~nm}$ thickness of silver at top plate; b 3DCLH with $37 \mathrm{~nm}$ thickness of silver at top plate
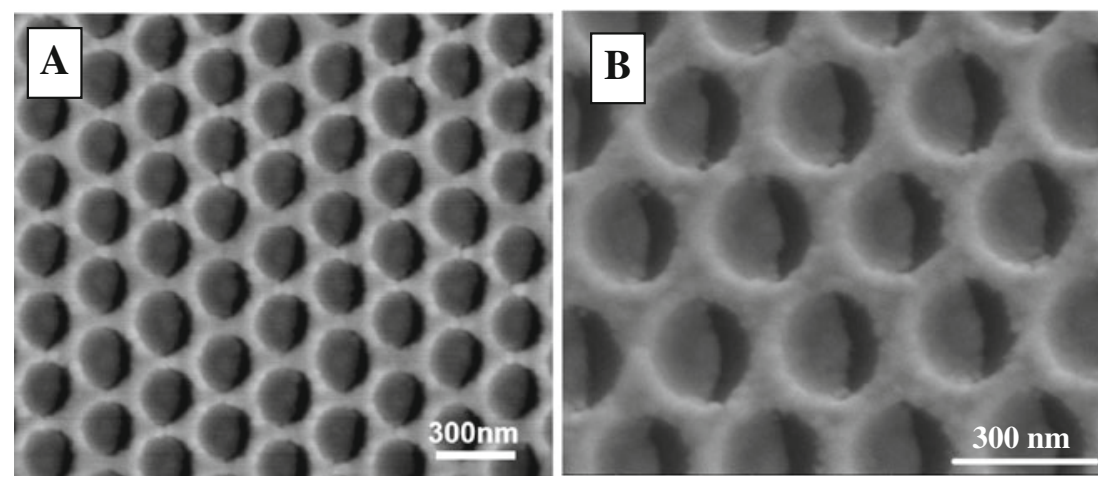
Fig. 3 a The zero-order angleresolved transmission spectra of the 3DCLHs array shown in Fig. 2a for the electric field of incident p-polarized waves being along the cross-line; the curves have been plotted with subsequent vertical offsets of 5\%. The black thin line is the transmission of a thin silver film with a thickness of $55 \mathrm{~nm}$. The inserted red-white circles denote the electrical field of the incident beam, where red color indicates silver parts in the dimple, while white indicates the parts without silver. b The simulated and experimental transmission spectra in normal incidence

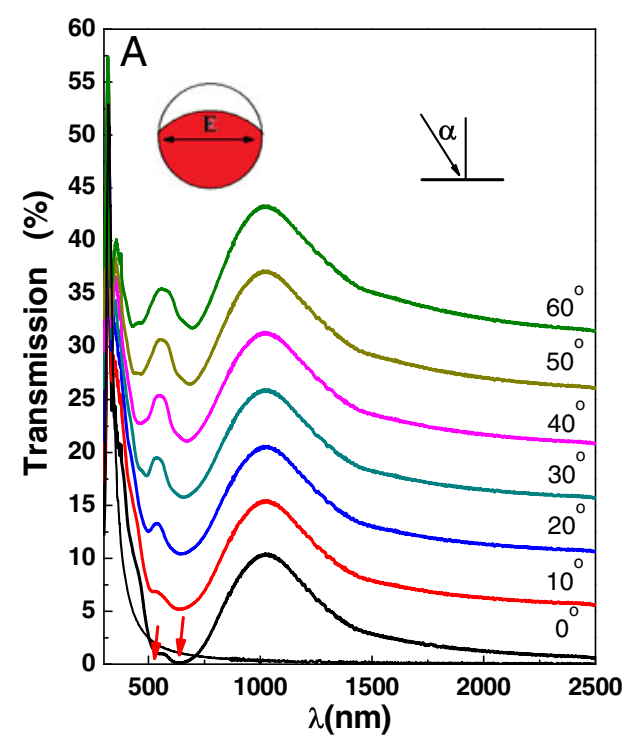

the crescent structure and rather than a periodic effect. At the dip position of $668 \mathrm{~nm}$, the electric field is mainly trapped within the dimples covered with silver, and thus, most of which is reflected back into the air. Because the electric field is localized at the $3 \mathrm{D}$ crescent-like holes area, the peak at $962 \mathrm{~nm}$ and dip at $668 \mathrm{~nm}$ are caused by localized surface plasmon. This can also be found from the experimental angle-resolved transmission spectra of Fig. 3a, where the transmission peak position at $1,028 \mathrm{~nm}$ and dip position at $634 \mathrm{~nm}$ do not change with the change of incident angle. While for the peak position at $600 \mathrm{~nm}$ and dip position at $554 \mathrm{~nm}$ will change their positions with the change of the incident angle, this can be found from their electric field distribution which is weakly localized at dip's area, especially the area having silver. Their field distribu- tions also indicate dipole resonance as well. This is caused by the combination of surface plasmon polariton and localized surface plasmon, the detailed mechanism becomes more complicated which requires further study.

Figure 5a represents the angle-resolved zero-order transmission when the electric field of the incident $p$ polarized waves is perpendicular to the cross-line of 3DCLH. Because the unit cell of the 3DCLHs array has no mirror symmetry for positive and negative incident angles, we measured the angle-resolved spectra from $-60^{\circ}$ to $60^{\circ}$ as shown in the inset. There exists a wide dip at $595 \mathrm{~nm}$ when the incident angle is $0^{\circ}$. The peak transmission at normal incidence at the wavelength of $1,336 \mathrm{~nm}$ is about $32.22 \%$ which is much higher than that in the case of electric field being along with the cross-line
Fig. 4 The electric field amplitude at the plane of $z=-20 \mathrm{~nm}$ when the cross-line of the 3DCLH oriented to $\Gamma-K$ for the electric field of the p-polarized waves parallel to the cross-line of the 3DCLH. a $\lambda=952$, $\mathbf{b} \lambda=$ 668, c $\lambda=600$, and $\mathbf{d} \lambda=554 \mathrm{~nm}$. The electric field amplitude distribution for the cross-line of the 3DCLH oriented to $\Gamma-M$ are nearly the same. The plus and minus signs indicate the polarity of the charge
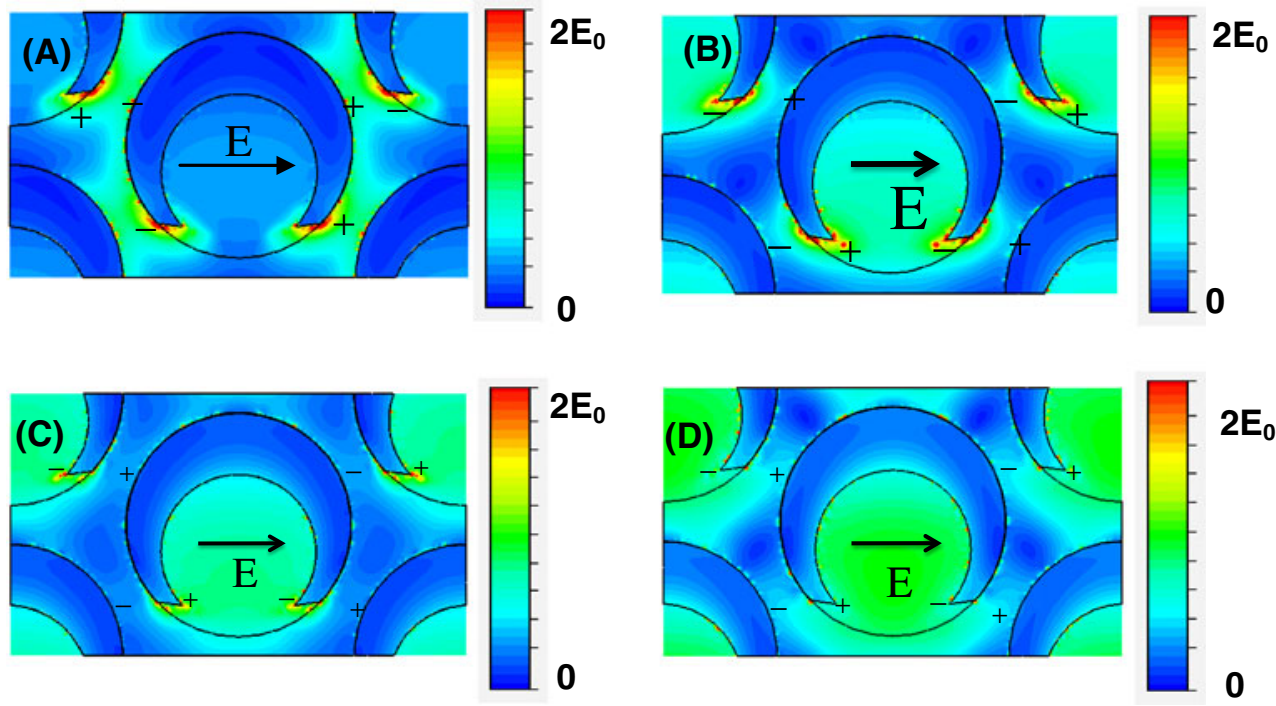
Fig. 5 a and $\mathbf{b}$ are the zeroorder angle-resolved transmission spectra of the 3DCLHs array shown in Fig. 2a for the electric field being perpendicular to the cross-line of 3DCLH, $\mathbf{a}$ for p-polarized waves; $\mathbf{b}$ for spolarized waves. In all spectra graphs, the curves have been plotted with subsequent vertical offsets of 5\%. The black thin line in (a) is the transmission of a thin silver film with a thickness of $55 \mathrm{~nm}$. The inserted redwhite circles denote the electrical field of the incident beam, where red color indicates silver parts in the dimple, while white indicates the parts without silver. c The simulated and experimental transmission of 3DCLH's at normal incidence when the electric field is perpendicular to the cross-line of the 3DCLHs; $d$ the electric field amplitude distribution at the wavelength of $1,442 \mathrm{~nm}$ in peak transmission at Fig. 5c
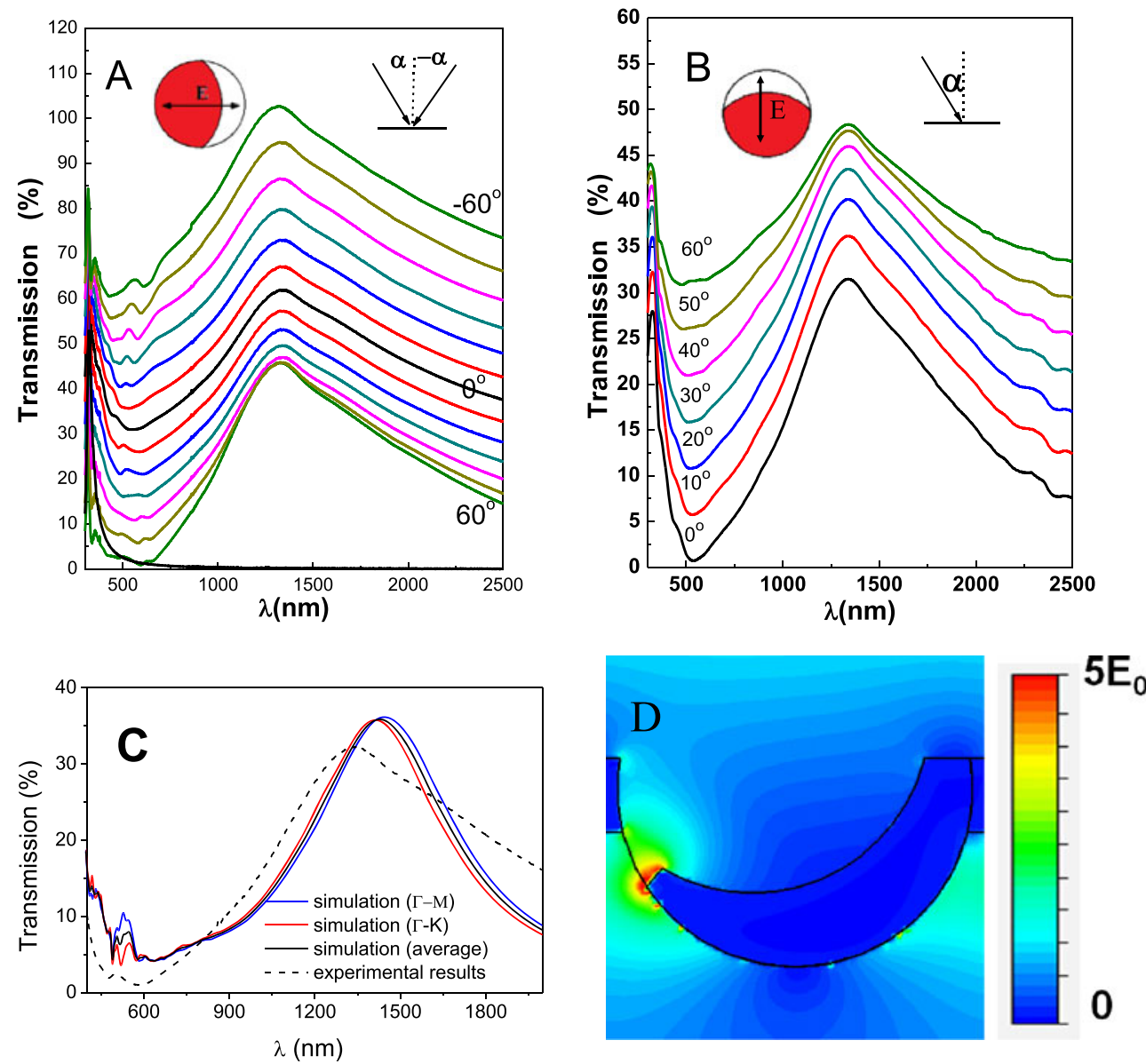

of 3DCLH. If the transmission is normalized with the fraction of holes' area of $7.7 \%$, the transmission can be over 4.18, which exhibits an extraordinary optical transmission phenomenon. It is surprising that this transmission peak does not shift with the change of incident angle. To understand the physics of this transmission, we also simulate the transmission through FDTD method. It is shown in Fig. 5c, where the simulated transmission is an average of the transmissions of the 3DCLHs arrays when the cross-lines of 3DCLHs are oriented to $\Gamma-M$ and $\Gamma-K$, respectively. The transmission spectra of the 3DCLHs arrays with different orientations of the 3DCLH's crossline are shown in this figure. It is found that the transmission spectra of these two cases with different orientations of the cross-lines are nearly the same. This means that the orientation of the cross-line of the 3DCLH does not affect the transmission in the normal incidence. Comparing the simulating results with the experimental results, it is found that the shape of the transmission spectra agrees well with each other except that the position of the peak has slightly deviation where the simulating and experimental transmission peaks are at 1,442 and $1,336 \mathrm{~nm}$, respectively. The deviation is caused by the simulation structure is not perfectly matching to the

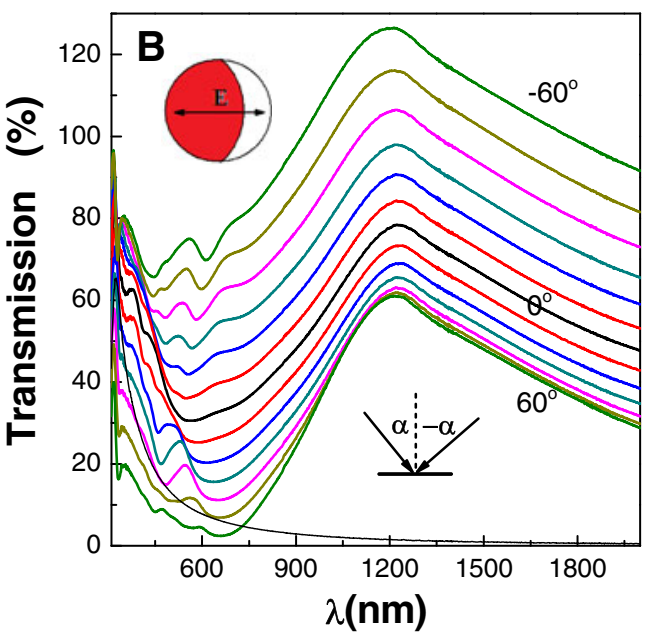

Fig. 6 The angle-resolved transmission spectra of the 3DCLHs array shown in Fig. $2 b$ for the electric field of the incident p-polarized waves being vertical to the cross-line, where the black thin line is the transmission of a thin silver film with a thickness of $37 \mathrm{~nm}$; the curves have been plotted with subsequent vertical offsets of $5 \%$. The inserted red-white circle denotes the electrical field of the incident beam, where red indicates silver parts in the dimple, while white indicates the parts without silver 
experimental one as mentioned before. For the wavelength shorter than $600 \mathrm{~nm}$, it is not considered here because both SPP and LSP play roles in the transmission and makes it more complicated. Looking into the electric field distribution at the peak position shown in Fig. 5d, it can be found that the electric field is strongly localized at the low arc of the 3DCLH, which indicates an asymmetric localized surface plasmon resonance. Such a resonance, it could show an angle insensitive coupling to incident light similar to the symmetric localized plasmon resonance [27], this might be the reason why the peak is consistent with the change of the incident angle when the electric field of the incident beam is perpendicular to the cross-line of the 3DCLH. Therefore, we found a novel physical phenomenon of EOT whose peak position is determined by an asymmetric LSP rather than SPP. The peak position is nearly stable with the change of incident angles. It might be useful for the sub-wavelength integrated optics and optical sensors.

Because SPP can only exist efficiently in the p-polarized wave and does not exist in s-polarized waves [42] to further exclude the SPP effect on the abnormal EOT, we also measured the zero-order transmission when the electric field of the incident s-polarized waves is perpendicular to the cross-line of the 3DCLH, as shown in Fig. 5b. It is found that, even with the incident angle up to $60^{\circ}$, the transmission is still as high as $18.34 \%$, and the peak position does not shift as well. Therefore, we could also obtain a conclusion that this EOT is induced by LSP rather than SPP.

To study this EOT in details, we fabricated various 3DCLHs arrays with different thicknesses of the silver film and various gaps. Figure 6 shows the p-polarized angle-resolved transmission of the 3DCLHs array with $37 \mathrm{~nm}$ thickness of silver as shown in Fig. 2b. The filling fraction of the crescent-like holes is $13.56 \%$. For the electric field of the incident waves being perpendicular to the cross-line, there also exists an EOT peak $(48.32 \%)$ at the wavelength of $1,228 \mathrm{~nm}$ which does not change with the incident angle, if it is normalized with the fraction of the holes' area, the transmission can reach 3.56. It is found that, if the hole area is nearly the same, the peak position shifts to the short wavelength with the increase of the thickness of the silver; if the thicknesses of the silver are nearly the same, the peak position shifts to the long wavelength with the decrease of the gap, and the normalized transmission with the fraction of the hole area is also becoming larger with the decrease of the gap.

\section{Conclusion}

In summary, we have developed a technique to fabricate periodic arrays of three-dimensional crescent-like holes based on an inverted colloidal lithography method and shadow metal evaporation. The shape of 3DCLH can be adjusted via varying the evaporation angle. The optical measurements show that, there exists a shape-dependent EOT peak for the electric field of the incident beam perpendicular to the cross-line of the 3DCLH. Especially, this transmission peak position does not change with the incident angle, which is different from the previous reports. Experiments and simulations show that this EOT is dominated by an asymmetric localized surface plasmon resonance induced by $3 \mathrm{DCLH}$. This might be valuable for the optical sensing and optical integrated circuits.

Acknowledgments Authors thank Prof. Che-Ting Chan and Prof. Zhao-Qing Zhang from Hong Kong University of Science of Technology with their fruitful discussion. This work is supported by the following projects: NNSFC (10774195, U0834001, 10974263, 10725420, and U0934002), Ministry of Education (grant 309024), Program for New Century Excellent Talents in University, and National Basic Research Program of China under grant no. 2010 CB923200.

Open Access This article is distributed under the terms of the Creative Commons Attribution Noncommercial License which permits any noncommercial use, distribution, and reproduction in any medium, provided the original author(s) and source are credited.

\section{References}

1. Ebbesen TW, Lezec HJ, Ghaemi HF, Thio T, Wolff PA (1998) Nature 391:667

2. Genet C, Ebbesen TW (2007) Nature 445:39

3. DiMaio JR, Ballato J (2006) Opt Express 14:2380

4. Shinada S, Hashizume J, Koyama F (2003) Appl Phys Lett 83:836

5. Hashizume J, Koyama F (2004) Appl Phys Lett 84:3226

6. Guo BS, Song GF, Chen LH (2007) Appl Phys Lett 91:2

7. Garcia-Vidal FJ, Martin-Moreno L, Ebbesen TW, Kuipers L Reviews of Modern Physics 82:729

8. Dahlin A, Zach M, Rindzevicius T, Kall M, Sutherland DS, Hook F (2005) J Am Chem Soc 127:5043

9. Brolo AG, Gordon R, Leathem B, Kavanagh KL (2004) Langmuir 20:4813

10. Lesuffleur A, Kumar LKS, Brolo AG, Kavanagh KL, Gordon R (2007) J Phys Chem C 111:2347

11. Degiron A, Lezec HJ, Barnes WL, Ebbesen TW (2002) Appl Phys Lett 81:4327

12. Degiron A, Ebbesen TW (2005) J Opt A-Pure Appl Opt 7:S90

13. Koerkamp KJK, Enoch S, Segerink FB, van Hulst NF, Kuipers L (2004) Phys Rev Lett 92:18

14. Ruan ZC, Qiu M (2006) Phys Rev Lett 96:23

15. Liu HT, Lalanne P (2008) Nature 452:728

16. Gordon R, Brolo AG, McKinnon A, Rajora A, Leathem B, Kavanagh KL (2004) Phys Rev Lett 92:3

17. Garcia-Vidal FJ, Moreno E, Porto JA, Martin-Moreno L (2005) Phys Rev Lett 95:10

18. Orbons SM, Roberts A (2006) Opt Express 14:12623

19. Bao YJ, Peng RW, Shu DJ, Wang M, Lu X, Shao J, Lu W, Ming NB (2008) Phys Rev Lett 101:8 
20. Wu S, Wang QJ, Yin XG, Li JQ, Zhu D, Liu SQ, Zhu YY (2008) Appl Phys Lett 93:101113

21. Wang C, Gu J, Han J, Xing Q, Tian Z, Liu F, Chai L, Li Y, Hu M, Wang Q (2010) Appl Phys Lett 96:251102

22. Mary A, Rodrigo SG, Martin-Moreno L, Garcia-Vidal FJ (2007) Phys Rev B 76:19

23. Zhan P, Wang ZL, Dong H, Sun J, Wu J, Wang HT, Zhu SN, Ming NB, Zi J (2006) Adv Mater 18:1612

24. Marani R, Marrocco V, Grande M, Morea G, D’Orazio A, Petruzzelli V (2011) Plasmonics 6:469

25. Hou Y (2011) Plasmonics 6:289

26. Rui G, Zhan Q, Ming H (2011) Plasmonics 6:521

27. Rodrigo SG, Mahboub O, Degiron A, Genet C, Garcia-Vidal FJ, Martin-Moreno L, Ebbesen TW (2010) Opt Express 18:23691

28. Yang JC, Gao HW, Suh JY, Zhou W, Lee MH, Odom TW (2010) Nano Lett 10:3173

29. Pang Y, Genet C, Ebbesen TW (2007) Opt Commun 280:10

30. Shumaker-Parry JS, Rochholz H, Kreiter M (2005) Adv Mater $17: 2131$

31. Zhu FQ, Fan DL, Zhu XC, Zhu JG, Cammarata RC, Chien CL (2004) Adv Mater 16:2155
32. Bukasov R, Shumaker-Parry JS (2007) Nano Lett 7:1113

33. Fredriksson H, Alaverdyan Y, Dmitriev A, Langhammer C, Sutherland DS, Zaech M, Kasemo B (2007) Adv Mater 19:4297

34. Prikulis J, Hanarp P, Olofsson L, Sutherland D, Kall M (2004) Nano Lett 4:1003

35. Kosiorek A, Kandulski W, Glaczynska H, Giersig M (2005) Small $1: 439$

36. Hulteen JC, Van Duyne RP (1995) J Vac Sci Technol A: Vac, Surf, Films 13:1553

37. Jang SG, Choi DG, Heo CJ, Lee SY, Yang SM (2008) Adv Mater 20:4862

38. Chen ZM, Gang T, Yan X, Li X, Zhang JH, Wang YF, Chen X, Sun ZQ, Zhang K, Zhao B, Yang B (2006) Adv Mater 18:924

39. Heo CJ, Kim SH, Jang SG, Lee SY, Yang SM (2009) Adv Mater 21:1726

40. Xu HX, Rao WY, Meng J, Shen Y, Jin CJ, Wang XH (2009) Nanotechnology 20:46

41. Berenger JP (1996) J Comput Phys 127:363

42. Zayats AV, Smolyaninov II, Maradudin AA (2005) Phys Rep 408:131 